\title{
Extremaleigenschaften von Kraftkonstanten
}

III. Über einen Zusammenhang des "progressive rigidity model" mit Extremaleigenschaften von Kraftkonstanten der Schwingungsbewegung von Molekülen

\author{
G. STREY \\ Sektion Physik der Universität München \\ (Z. Naturforsch. 24 a, 729-730 [1969] ; eingegangen am 15. Februar 1969)
}

\begin{abstract}
Es wird der Beweis geführt, daß das Modell der progressiven Starrheit von Torkington mit sukzessive eingeführten Minimalforderungen an die diagonalen Elemente der Matrix der Kraftkonstanten verknüpft ist.
\end{abstract}

In der letzten Zeit sind in mehreren Arbeiten ${ }^{1-8}$ Extremaleigenschaften von Kraftkonstanten der Schwingungsbewegung von Molekülen diskutiert worden. Freeman ${ }^{5}$, sowie Peacock und Müller ${ }^{6}$ haben dabei darauf aufmerksam gemacht, da $\beta$ im Falle einer Symmetrierasse mit zwei Schwingungsmoden $(n=2)$ die Extremalforderung

$$
F_{22}=\operatorname{Min} F_{22}(\varphi)
$$

identisch ist mit dem schon früher von TORKINGTON ${ }^{9}$ und LARNAUdiE ${ }^{10}$ vorgeschlagenen Modell der progressiven Starrheit: "progressive rigidity model". Noch um einiges früher haben jedoch russische Autoren ${ }^{11,12}$ das gleiche Modell als 0-te Näherung zur Berechnung von Kraftkonstanten benutzt und auch in der Folgezeit an zahlreichen Beispielen diskutiert ${ }^{13-17}$.

Es soll in dieser Arbeit gezeigt werden, daß das Modell der progressiven Starrheit nicht nur im Spezialfall $n=2$, sondern auch im allgemeinen Falle von $n$ Schwingungsmoden in einer Symmetrierasse mit bestimmten Extremalforderungen an die Diagonalelemente $F_{i i}$ der Kraftkonstantenmatrix $\boldsymbol{F}$ zusammenhängt.

Die Säkulargleichung der Schwingungsbewegung eines Moleküls lautet in der Notation von WiLson,

1 G. Strey, Raman-Kolloquium, Magdeburg 1966; J. Mol. Spectry 24, 87 [1967].

2 H. J. Becher u. K. Ballein, Z. Phys. Chem. Frankfurt 54, 302 [1967].

3 G. Strey u. K. Klauss, Frühjahrstagung der DPG, Augsburg 1967; Z. Naturforsch. 23 a, 1717 [1968].

4 G. Strey u. K. Klauss, Z. Naturforsch. 23 a, 1667 [1968].

5 D. E. Freeman, J. Mol. Spectry. 27, 27 [1968].

6 C. J. Peacock u. A. Müller, J. Mol. Spectry. 26, 454 [1967] ; Z. Naturforsch. 23 a, 1029 [1968].

7 A. Müller, Z. Phys. Chem. Leipzig 238, 116 [1968].

8 D. E. Freeman, Chem. Phys. Letters 2, 615 [1968].

9 P. Torkington, J. Chem. Phys. 17, 1026 [1949].

10 M. Larnaudie, J. Phys. Radium 15, 365 [1954].

11 M. A. Eliashevich, Zh. Fiz. Khim. 15, 847 [1941].

\section{Decius und Cross ${ }^{18}$}

$$
\text { ( } \boldsymbol{G} \boldsymbol{F}) \mathbf{L}=\mathbf{L} \boldsymbol{\Lambda},
$$

wobei die Eigenvektormatrix $\boldsymbol{L}$ durch die Gleichung

$$
\mathbf{L} \tilde{\mathbf{L}}=\boldsymbol{G}
$$

normiert ist. Die Eigenwerte $\lambda_{i}$ der Säkularmatrix $(\boldsymbol{G} \boldsymbol{F})$, zusammengefaßt in der Diagonalmatrix $\boldsymbol{\Lambda}$, seien fallend geordnet

$$
\lambda_{1}>\lambda_{2}>\ldots>\lambda_{n}>0 \text {. }
$$

Mit der Bezeichnung $\boldsymbol{A}=\boldsymbol{L}^{-1}$

$$
\text { folgt dann } \boldsymbol{F}=\tilde{\boldsymbol{A}} \boldsymbol{\Lambda} \boldsymbol{A}
$$

und

$$
\boldsymbol{G}^{-1}=\tilde{\boldsymbol{A}} \boldsymbol{A} .
$$

Das Modell der progressiven Starrheit beruht in dieser Notation darin ${ }^{9,13}$, die Matrix $\mathbf{L}$ und damit auch die Matrix $\boldsymbol{A}$ in der Form einer unteren Dreiecksmatrix anzunehmen.

Wegen Gl. (5) kann man die Diagonalelemente $F_{i i}$ von $\boldsymbol{F}$ als Funktionen der jeweils $n$ Elemente $a_{k i}(k=1,2, \ldots, n)$ der $i$-ten Spalte von $\boldsymbol{A}$ ansehen. Diese Elemente erfüllen jedoch wegen Gl. (6) noch eine bestimmte Anzahl von Nebenbedingungen. Strey und Klauss ${ }^{3}$ haben gezeigt, daß, wenn die Kraftkonstante $F_{n n}$ unter der Nebenbedingung

$$
a_{1 n}^{2}+a_{2 n}^{2}+\ldots+a_{n n}^{2}=\left(G^{-1}\right)_{n, n}
$$

12 M. V. Volkenshtein, M. A. Eliashewich u. B. I. StepaNov, „Kolebania molekul“, Bd. 1, Gittl, Moskau 1949.

13 I. V. ORlova u. I. N. GodNEv, Opt. Spectry. USSR 6, 284 [1959].

14 I. N. Godnev, A. M. Aleksandrovskaya u. I. V. Rigina, Opt. Spectry. USSR 7, 172, 495 [1959].

15 A. M. Aleksandrovskaya u. I. N. Godnev, Opt. Spectry. USSR 9, 144 [1960]; 10, 14 [1961].

16 V. N. Vinogradova u. I. N. Godnev, Izv. Vysshikh. Uchebn. Zavedenii 1965, Nr. 1, S. 57; Opt. Spectry. USSR Suppl. 2, 40 [1966].

17 A. M. Aleksandrovskaya, V. N. Vinogradova u. I. N. Godnev, Opt. i Spektroskopiya, Suppl. 3, 64. [1967].

18 E. B. Wilson, J. C. Decius u. P. C. Cross, Molecular Vibrations, McGraw-Hill, New York 1955. 
ihren minimalen Wert annimmt, die Elemente $a_{j n}$ der $n$-ten Spalte von $\boldsymbol{A}$ durch die Gleichungen

$$
\begin{aligned}
& a_{j n}=0 \quad \text { für } j \neq n, \\
& a_{n n}=\left(G^{-1}\right)_{n, n}^{1 / 2}
\end{aligned}
$$

gegeben sind. Wegen Gl. (6) gilt dann aber

$$
a_{n, n-1}=\left(\mathbf{G}^{-1}\right)_{n-1, n}\left(\mathbf{G}^{-1}\right)_{n, n}^{1 / 2},
$$

während die restlichen Elemente $a_{j, n-1} \quad(j=1,2$, $\ldots, n-1) \operatorname{der}(n-1)$-ten Spalte von $\boldsymbol{A}$ noch freie Variablen sind. Faßt man nun die Kraftkonstante $F_{n-1, n-1}$ als Funktion dieser $n-1$ Variablen auf, $F_{n-1, n-1}=\left(\lambda_{1} a_{1, n-1}^{2}+\lambda_{2} a_{2, n-1}^{2}\right.$

$$
\left.+\ldots+\lambda_{n-1} a_{n-1, n-1}^{2}\right)+\lambda_{n} a_{n, n-1}^{2}
$$

und berücksichtigt zusätzlich die Nebenbedingung

$$
\begin{gathered}
\Phi_{n-1}=\left(\boldsymbol{G}^{-1}\right)_{n-1, n-1}-\left(\boldsymbol{G}^{-1}\right)_{n-1, n}^{2} /\left(\mathbf{G}^{-1}\right)_{n, n} \\
-\left(a_{1, n-1}^{2}+a_{2, n-1}^{2}+\ldots+a_{n-1, n-1}^{2}\right)=0,(10)
\end{gathered}
$$

so lautet die Extremalforderung

$$
\frac{\partial f_{n-1}}{\partial a_{j, n-1}}=0 \quad(j=1,2, \ldots, n-1),
$$

worin $f_{n-1}=F_{n-1, n-1}+\varrho \Phi_{n-1}$ und $\varrho$ der LagrangeMultiplikator ist. Daraus folgt das Gleichungssystem

$$
a_{j, n-1}\left(\lambda_{j}-\varrho\right)=0 \quad(j=1,2, \ldots, n-1) .
$$

Wäre hierin $\varrho \neq \lambda_{j}$ für alle $j$, so würde $a_{j, n-1}=0$ für alle $j$ folgen. Das kann aber nicht sein, weil dann aus Gl. (6)

$$
a_{n, n-1}^{2}=\left(\mathbf{G}^{-1}\right)_{n-1, n-1}
$$

folgen würde, was mit Gl. (8) zur Folge hätte, daß der Hauptminor $M_{n-1, n}$, der aus $\mathbf{G}^{-1}$ entsteht, wenn man alle Zeilen und Spalten bis auf die $(n-1)$-te und die $n$-te streicht, verschwindet. Die Matrix $\boldsymbol{G}^{-1}$ ist aber positiv definit ${ }^{18}$ und hat daher nur positive Hauptminoren.

Das Gleichungssystem (12) hat daher nur die Lösungen

$$
\begin{gathered}
\varrho=\lambda_{k} \\
a_{j, n-1}=0 \quad \text { für } \quad j \neq k \\
a_{k, n-1}^{2}=\left(\left(\boldsymbol{G}^{-1}\right)_{n-1, n-1} \cdot\left(\mathbf{G}^{-1}\right)_{n, n}-\right. \\
\left.-\left(\mathbf{G}^{-1}\right)_{n-1, n}^{2}\right) /\left(\boldsymbol{G}^{-1}\right)_{n, n}, k=1,2, \ldots, n-1 .
\end{gathered}
$$

Die stationären Lösungen für $F_{n-1, n-1}$ ergeben sich daraus zu

$$
\begin{gathered}
F_{n-1, n-1}=\lambda_{k} a_{k, n-1}^{2}+\lambda_{n} a_{n, n-1}^{2}, \\
k=1,2, \ldots, n-1 .
\end{gathered}
$$

Seinen Minimalwert nimmt die Kraftkonstante $F_{n-1, n-1}$ infolgedessen an, wenn $k=n-1$ gesetzt wird. Dann ist

$$
\begin{aligned}
a_{j, n-1} & =0 \text { für } j=1,2, \ldots, n-2, \\
a_{n-1, n-1} & =\left(M_{n-1, n} / M_{n}\right)^{1 / 2} .
\end{aligned}
$$

Hierin soll $M_{n-j, n-j+1, \ldots, n} \quad(j=1,2, \ldots, n-1)$ den Hauptminor der Matrix $\boldsymbol{G}^{-1}$ bedeuten, der entsteht, wenn man in $\boldsymbol{G}^{-1}$ die Zeilen und Spalten $1,2, \ldots, n-j-1$ herausstreicht. Das Vorzeichen von $a_{n-1, n-1}$ darf positiv gewählt werden, weil die Eigenvektoren der Säkulargleichung nur bis auf ihr Vorzeichen festgelegt sind.

Man kann auf diese Weise fortfahren. Die Elemente $a_{n-1, n-2}$ und $a_{n, n-2}$ der $(n-2)$-ten Spalte von $\boldsymbol{A}$ sind auf Grund des bisherigen und der Gl. (6) bereits bestimmt. Die übrigen können hingegen wieder durch eine Minimalforderung an die Kraftkonstante $F_{n-2, n-2}$,

$$
\begin{aligned}
F_{n-2, n-2} & =\left(\lambda_{1} a_{1, n-2}^{2}+\ldots+\lambda_{n-2} a_{n-2, n-2}^{2}\right) \\
& +\lambda_{n-1} a_{n-1, n-2}^{2}+\lambda_{n} a_{n, n-2}^{2},
\end{aligned}
$$

unter der Nebenbedingung

$$
\begin{gathered}
\left(a_{1, n-2}^{2}+a_{2, n-2}^{2}+\ldots+a_{n-2, n-2}^{2}\right)+a_{n-1, n-2}^{2} \\
+a_{n, n-2}^{2}=\left(\mathbf{G}^{-1}\right)_{n-2, n-2}
\end{gathered}
$$

festgelegt werden. Es ergibt sich daraus

$$
\begin{aligned}
a_{j, n-2} & =0 \quad \text { für } j<n-2, \\
a_{n-2, n-2} & =\left(M_{n-2, n-1, n} / M_{n-1, n}\right)^{1 / 2},
\end{aligned}
$$

wobei das Vorzeichen von $a_{n-2, n-2}$ wieder positiv gewählt werden darf.

Sukzessive können auf diese Weise durch Minimalforderungen an die diagonalen Kraftkonstanten $F_{i i}$ alle Elemente der Matrix $\boldsymbol{A}$ derart bestimmt werden, daß eine untere Dreiecksmatrix entsteht.

Herrn Prof. Dr. J. Brandmüller danke ich für sein Interesse an dieser Arbeit. 